\title{
Consistency Priorities for Multi-device Design
}

\author{
Rodrigo de Oliveira and Heloísa Vieira da Rocha \\ Campinas State University, Institute of Computing, Campinas SP 13081-970, Brazil \\ oliveira@ic.unicamp.br, heloisa@ic.unicamp.br
}

\begin{abstract}
We propose consistency priorities to support multi-device interface design minimizing the user's cognitive effort while performing the same task on different interfaces. The methodology is being evaluated through a framework that generates Pocket PC interfaces from desktop web pages. Initial results point to the acceptance of the approach.
\end{abstract}

\section{Introduction}

Mobile devices introduced a great challenge for Human Computer Interaction: to develop multi-device interfaces for today's applications. Some have tried device oriented designs with linear transformations, creating mobile interfaces from scratch, like Avantgo (www.avantgo.com) and Usable Net (www.usablenet. com); others looked for dynamic and automatic adaptations, but still focusing on the device 138 . These and other related approaches were well received, but the generated interfaces are different from the original in some aspects that complicate interaction with more than one device to perform the same task, especially when refinding and/or comparing information [79. Many works addressed consistency and continuity problems focusing on user interface generation [45] and task migration [11, but their guidelines are generally not sufficiently concrete for an automatic interface framework. A recent proposal [10] solves the multidevice design problem by passing the control of every appliance to a handheld interface generated automatically. Despite the valued ideas, many device specific interaction types important to each context of use can be lost on the process, besides the need to carry a mobile device to control everything.

We propose consistency priorities for multi-device interface design that aims to improve usability and the user's experience when performing similar tasks on different devices. Some prototypes were implemented for automatic desktop web page adaptation to handhelds, such as Pocket PCs and smartphones. Initial evaluations point to the acceptance of this approach. Formal user evaluations will be conducted to check these first impressions.

\section{Consistency Priorities Proposal}

Pyla et al. [11] argue that consistency needs to be better defined if it is to be the overriding factor in the design of multiple user interfaces. In fact, there isn't a consensus about what consistency really is and how it can be applied [10]. 
We think about consistency on the user's side. Individuals mentally draw conclusions about objects or events on the basis of previous observations of similar objects or events. These internal constructions that can be manipulated enabling predictions are called mental model [2]. Figure 1 sketches the user's mental model update cycle while executing tasks.

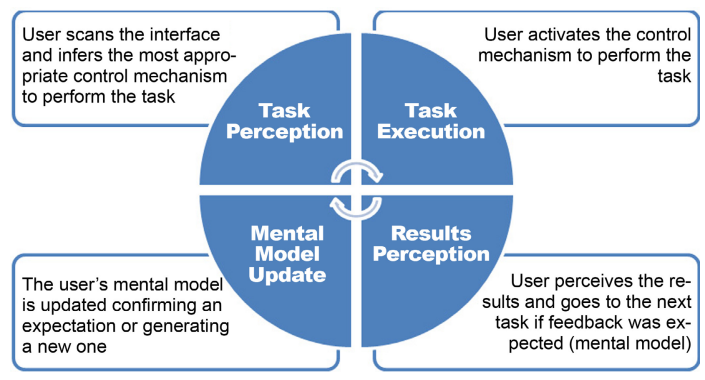

Fig. 1. User's mental model update cycle. Task perception and execution are the key processes to build a consistent mental model for decision making.

In order to help users form an accurate and useful mental model of a system while interacting with any of its interfaces, we suggest applying consistency on multi-device contexts using the following priorities:

1. Task Perception - the same control mechanisms to execute a task and their disposal on the interface. If these requirements cannot be followed with good usability on devices with different control attributes (e.g. size, weight, material, etc.) and properties (e.g. fluidity, flexibility, opacity, etc.), perception should be mapped to each device's interaction type maintaining usability.

2. Task Execution - the same actions flow to execute a task. If the control mechanisms available on a given interface had to be adapted for the others by the task perception priority, the actions flow should be maintained on a logical perspective. Although this may repass bad design decisions and lose opportunity to improve usability on each interface independently, user's decision making is supported under a consistent multi-device context, providing ease of learning/remembering and safety of use. Next priority improves efficiency.

3. Task Personalization - the ability to change task perception and execution according to the users' preferences. The goal is to achieve the best design for any user which is the configuration that user expects. This can be related to the personally consistent design concept [10, but with an active position for the user. As a result, efficiency and ease of expert use is provided to avoid the downsides of consistency [6].

It's important to understand the correct application of these consistency priorities as they can be easily misunderstood. For example, if an individual wants to check an account balance through an ATM machine, a tablet PC and a telephone, there is no possibility to perceive and perform the task in the same way. If 
the mentioned devices are important to the end user, adaptation to the contrasting interaction types is a price they are willing to pay. In fact, that's part of the task personalization priority. So the focus is to provide the same task perception and execution under a logical perspective, be it through words typed, written or said. This is in accordance with Nichols' work 10] about benefitting from user's experience, but opens space for the rich interaction types of the actual appliances in a consistent way.

\section{Towards an Empirical Validation}

On this section, we are going to take an application designed for multi-device access and improve it using our proposal. The application chosen is the Summary Thumbnail [7], a prototype designed to automatically adapt desktop web pages for handhelds. Here's how it works: the original web page is shrunken to fit horizontally on the smaller screen, text font is increased to improve legibility and letters are cropped from right to left until sentences fit on the available space. Complete texts can be read by accessing the detailed view through a click on the page, which moves to the original desktop interface with full scrolling.

After applying the consistency priorities to Summary Thumbnail, we identified two improvements: a better summarization process to avoid producing links with the same label (task perception) and a smoother transition between thumbnail and detailed views (task execution). On the first prototype generation, we used focus-plus-context to provide a faster detailed view over the thumbnail. Full texts and normal sized images are presented inside a hint window whenever users point to the corresponding object on the page. They can even confirm the full text to stay on page and this information is stored for future accesses (task personalization). As the hint detailed view loses format attributes useful on iconic systems, we are developing the next prototype generation with the Direct Migration 9] (no transformation applied to the page) inside the hint window with a lower opacity value to improve context view.

Currently, the automatic interface adaptation doesn't require additional Internet traffic and takes less than two seconds to adapt a web page using the browser script interpreter. The hardware used was the HP iPAQ Pocket PC h2400 running Windows Mobile 2003 but could be any other with a CSS, DHTML and JavaScript compatible browser. Recently, the first generation prototype was informally tested on a few institutions with much better impressions than the awarded commercial solution by Opera (www . opera.com/products/mobile/ reviews). Figure 2 compares screens generated by both approaches.

\section{Conclusions}

The consistency priorities proposal aims to improve usability and the user's experience when performing similar tasks on different devices. The methodology is being tested through prototypes designed to automatically adapt desktop web interfaces for handheld screens. Informal evaluations revealed better impressions 

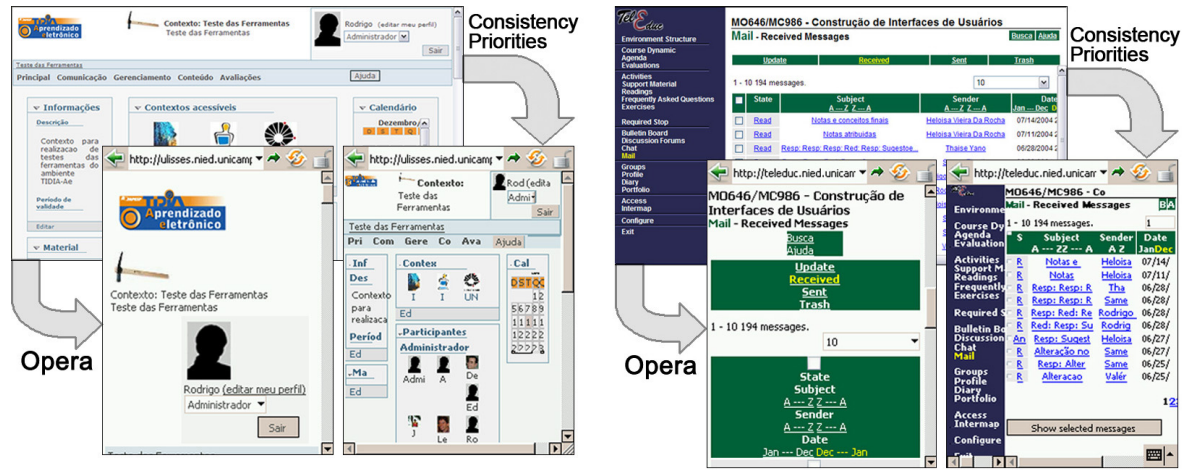

Fig. 2. Comparison between interfaces generated with the Consistency Priorities and Opera Fit to Screen. The first was much better evaluated on informal tests.

than a successful commercial approach. Next prototypes focus iconic interfaces and evaluations will be taken to verify the advantages of this proposal.

\section{References}

1. Berti, S., Correani, F., Mori, G., Paternò, F., Santoro, C.: Teresa: a transformationbased environment for designing and developing multi-device interfaces. In: Proc. CHI 2004 Extended Abstracts, Vienna, Austria, pp. 793-794 (April 2004)

2. Craik, K.J.W.: The nature of explanation. Cambridge University Press, Cambridge (1943)

3. Coninx, K., Luyten, K., Vandervelpen, C., Bergh, J.V.D., Creemers, B.: Dygimes: dynamically generating interfaces for mobile computing devices and embedded systems. In: Proc. MHCI 2003, Udine, Italy, pp. 256-270 (2003)

4. Denis, C., Karsenty, L.: Inter-usability of multi-device systems: A conceptual framework. In: Seffah, A., Javahery, H. (eds.) Multiple User Interfaces, pp. 373-385. John Wiley \& Sons, Chichester (2003)

5. Florins, M., Trevisan, D.G., Vanderdonckt, J.: The Continuity Property in Mixed Reality and Multiplatform Systems: A Comparative Study. In: CADUI 2004, Funchal, Portugal, pp. 323-334 (2004)

6. Grudin, J.: The case against user interface consistency. CACM 32(10), 1164-1173 (1989)

7. Lam, H., Baudisch, P.: Summary thumbnails: readable overviews for small screen web browsers. In: Proceedings of CHI 2005, Portland, OR, pp. 681-690 (2005)

8. Lin, J.: Using design patterns and layers to support the early-stage design and prototyping of cross-device user interfaces. Doctoral Thesis. Berkeley, California: University of California, p. 557 (2005)

9. Mackay, B., Watters, C., Duffy, J.: Web page transformation when switching devices. In: Proc. of the Mobile HCI 2004, Glasgow, Scotland, pp. 228-239 (2004)

10. Nichols, J.: Automatically generating high-quality user interfaces for appliances. Doctoral Thesis. Pittsburg, Pennsylvania: Carnegie Mellon University, pp. 322 (2006)

11. Pyla, P., Tungare, M., Pérez-Quiñones, M.: Multiple User Interfaces: Why consistency is not everything, and seamless task migration is key. In: Proceedings of the CHI 2006 Workshop on The Many Faces of Consistency in Cross-Platform Design. 